\title{
Comparison of Radical Cystectomy and Chemoradiotherapy in Patients with Locally Advanced Bladder Cancer
}

\author{
Masaomi Ikeda ${ }^{1}$, Kazumasa Matsumoto ${ }^{1 *}$, Morihiro Nishi ${ }^{1}$, Ken-ichi Tabata ${ }^{1}$, \\ Tetsuo Fujita ${ }^{1}$, Hiromichi Ishiyama ${ }^{2}$, Kazushige Hayakawa $^{2}$, Masatsugu Iwamura ${ }^{1}$
}

\begin{abstract}
The aim of this study was to evaluate the clinical outcomes of radical cystectomy (RC) and concurrent chemoradiotherapy (CRT) with methotrexate, vinblastine, doxorubicin, and cisplatin (MVAC) in patients with locally advanced bladder cancer (BC). From December 2000 to February 2012, 72 patients with locally advanced BC (T3-4a, N0 or N+, M0) received either RC or CRT. RC with bilateral pelvic lymph node dissection including the common iliac region as the standard procedure. Patients in the CRT group received one cycle of MVAC followed by radiotherapy with a half dose of MVAC and then two more cycles of MVAC. Standard fractionation at a daily dose of 1.8-2.0 Gy was used, with a median total dose of $50 \mathrm{~Gy}$ (range, 45-60 Gy). The 3-year progression-free survival (PFS) rates in the RC and CRT groups were 56.2\% and 25.6\%, respectively ( $p=-0.015)$ and the 3-year overall survival $(\mathrm{OS})$ rates were $63.5 \%$ and $48.1 \%(\mathrm{p}=\mathbf{0 . 2 7 2})$. Multivariate Cox proportional hazards regression analysis with application of a propensity score indicated that $\mathrm{RC}$ was a significant predictor of $\mathrm{PFS}(\mathrm{p}=\mathbf{0 . 0 3 3 )}$ but not of OS ( $p=0.291$ ). Among patients with locally advanced $B C$, PFS was significantly prolonged in the RC group compared with the CRT group. However, $\mathrm{RC}$ was not a significant predictor of OS. Although the sample size in this study was small, the results suggest that patient background and postoperative quality of life should be considered when choosing treatment strategy for locally advanced $\mathrm{BC}$.
\end{abstract}

Keywords: Radical cystectomy - chemoradiotherapy - locally advanced bladder cancer - survival

Asian Pac J Cancer Prev, 15 (16), 6519-6524

\section{Introduction}

In 2008 , the incidence of urothelial carcinoma (UC) of the bladder was approximately 386,300 and the disease was responsible for 150,200 deaths worldwide (Jemal et al., 2011). Although the majority of patients present with non-muscle-invasive bladder cancer, $20 \%-40 \%$ of patients with non-muscle-invasive bladder cancer eventually develop muscle invasive bladder cancer (MIBC). The survival rate is poor for MIBC; only approximately $45 \%$ of patients survive for 5 years regardless of the type of treatment (Grossman et al., 2003; Rodel et al., 2002; Stein et al., 2001). According to the European Urology Association and National Comprehensive Cancer Network guidelines, radical cystectomy (RC) with bilateral pelvic lymphadenectomy is the gold standard treatment for patients with MIBC (Stein et al., 2001; Hautmann et al., 2006). However, cisplatin-based systemic chemotherapy, such as a combination of methotrexate, vinblastine, doxorubicin and cisplatin (MVAC) (Sternberg et al., 1985) or a combination of gemcitabine and cisplatin (von der Maase et al., 2000), is the recommended standard treatment for inoperably advanced or metastatic UC.

Although multimodal treatment has become the standard of care for other kind of malignancies (Hashemi et al., 2013; Baykara et al., 2013; Liang et al., 2013; Kilic et al., 2012), chemoradiotherapy (CRT) is performed in an effort to preserve the bladder in patients with MIBC. Concurrent CRT has an advantage over external beam radiation therapy (EBRT) alone (Rodel et al., 2002; Efstathiou et al., 2012; Shipley et al., 1998; Yu et al., 2012), but only a few randomized trials have compared these two approaches to the treatment of MIBC (James et al., 2012; Coppin et al., 1996). Even in the absence of more-effective systemic therapy, improving bladder preservation treatments could provide patients with a choice of treatment and improve quality of life.

Although no randomized trial comparing RC and CRT has been conducted, evidence accumulated from several countries suggests that bladder preservation approaches may yield favorable results in selected patients (Rodel et al., 2002; Efstathiou et al., 2012; Shipley et al., 1998; James et al., 2012). We previously showed that MVAC combined with EBRT is effective in patients with locally 
advanced or metastatic bladder cancer (Ikeda et al., 2011). The aim of the current study was to evaluate the clinical outcomes of RC and CRT in patients with locally advanced bladder cancer.

\section{Materials and Methods}

\section{Patient eligibility and selection}

This study is a non-randomized retrospective study. The treatment modality was determined by consultation of a primary doctor and a patient. Therefore, this study is comparison of clinical outcomes of the patients who underwent either RC or CRT from December 2000 to February 2012. Among the 91 patients, 14 patients with muscle-invasive organ-confined disease (T2NOM0) and 5 patients who had received neoadjuvant systemic chemotherapy were excluded. The remaining 72 patients with locally advanced bladder cancer (T3-4a, N0 or N+, M0) were enrolled in the study.

The main inclusion criteria were as follows: Eastern Cooperative Oncology Group performance status (ECOG PS) of 0 to 2, according to World Health Organization criteria; white blood cell count of more than $3500 / \mu \mathrm{L}$; platelet count of more than $100,000 / \mu \mathrm{L}$; hemoglobin of more than $10 \mathrm{~g} / \mathrm{dl}$; hepatic function (serum bilirubin 1.5 $\mathrm{mg} / \mathrm{dl}$ or less); and renal function (serum creatinine 1.5 $\mathrm{mg} / \mathrm{dl}$ or less, and measured creatinine clearance of at least $60 \mathrm{ml} / \mathrm{min}$ ). Patients with nonmalignant systemic disease, including active infection, any clinically significant cardiac arrhythmia, or congestive heart failure, were not eligible. Written informed consent was obtained from all patients before treatment. The study was approved by the institutional review board at Kitasato University Hospital and conducted in accordance with the Declaration of Helsinki.

\section{Protocol design and treatment}

Thirty-two (44.4\%) patients received RC and bilateral lymphadenectomy. In males, standard RC involved resection of the bladder, prostate, seminal vesicles, and distal ureters; in females, an anterior pelvic exenteration included the removal of the bladder, entire urethra and adjacent vagina, uterus, and distal ureters. The urethra and urethra-supplying autonomous nerves were spared in cases in which an orthotopic neobladder was planned. Standard pelvic lymphadenectomy is defined as the removal of all lymphatic tissues around the common iliac, external iliac, internal iliac, and obturator region bilaterally. Urinary diversion was performed after RC as follows: neobladder in 9 patients, ileal conduit in 17, cutaneous ureterostomy in 5 and total urinary tract removal in 1.

Forty patients $(55.6 \%)$ received the CRT protocol that we described previously (MVAC combined with EBRT) (Ikeda et al., 2011). Briefly, after receiving one cycle of MVAC treatment, all patients received EBRT with a half dose of MVAC treatment followed by two more cycles of MVAC. Standard fractionation with a daily dose of 1.8-2.0 Gy was used, with a median total dose of $50 \mathrm{~Gy}$ (range, 45-60 Gy) over 5-6 weeks. Generally, the bladder tumor, bladder, and regional lymph nodes were treated by means of a four-field box technique.

\section{Treatment evaluation}

After RC, patients were generally followed up every 3-4 months for 2 years, every 6 months for the subsequent 3 years and annually thereafter. Follow-up included a physical examination, along with routine serum chemistry studies. Radiographic evaluation (with or without contrast-enhanced computerized tomography) of urinary diversion, the upper urinary tract and the local region, as well as a chest X-ray, were performed at 3 or 6 months postoperatively and annually thereafter. Bone scans and other radiographic evaluation were performed when clinically indicated.

During CRT treatment, blood cell count and serum chemistry analyses were carried out weekly, and creatinine clearance was calculated before MVAC treatment. Tumors were assessed by computerized tomography or magnetic resonance imaging every two cycles and responses were determined at least 4 weeks after administration. After CRT, cystoscopic surveillance and urine cytology occurred every 3-4 months for 2 years, every 6 months for the subsequent 3 years and annually thereafter.

On the basis of patient medical records, overall survival (OS) was measured from RC or CRT until death, and time to failure was measured until discontinuation of treatment, death, or progression. Patients were assigned to a response category according to the Response Evaluation Criteria in Solid Tumors guidelines (version 1.1). Adverse events were monitored according to the National Cancer Institute Common Terminology Criteria for Adverse Events (version 4.0).

\section{Statistical analysis}

Demographic, clinical, and pathological data were compared by means of chi-square tests and Mann-Whitney $\mathrm{U}$ tests. OS and progression-free survival (PFS) were analyzed by means of the Kaplan-Meier method, and differences between the groups were determined with the log-rank test. Multivariate analyses were performed with the Cox proportional hazards regression model, controlling for age, gender, ECOG PS, tumor stage, lymph node metastases, and treatment. In observational studies, the groups being compared often differ because of the lack of randomization and the retrospective nature of the studies.

In addition, if the number of events is small and there are multiple confounders, the estimates obtained from regression models can be incorrect. The propensity score is a good alternative to control imbalances between study groups when there are seven or fewer events per confounder variable (Cepeda et al., 2003). To calculate the propensity score for choosing RC, we used the following variables: age, gender, ECOG PS, tumor stage, and lymph node metastases. In addition to conventional multivariable analysis, a propensity score adjustment approach was performed to avoid overfitting. All analyses were performed with Stata software (ver. 11 for Windows, Stata, Chicago, IL, USA). A P value of $<0.05$ was considered to indicate statistical significance, and all $\mathrm{P}$ values are two-sided. 

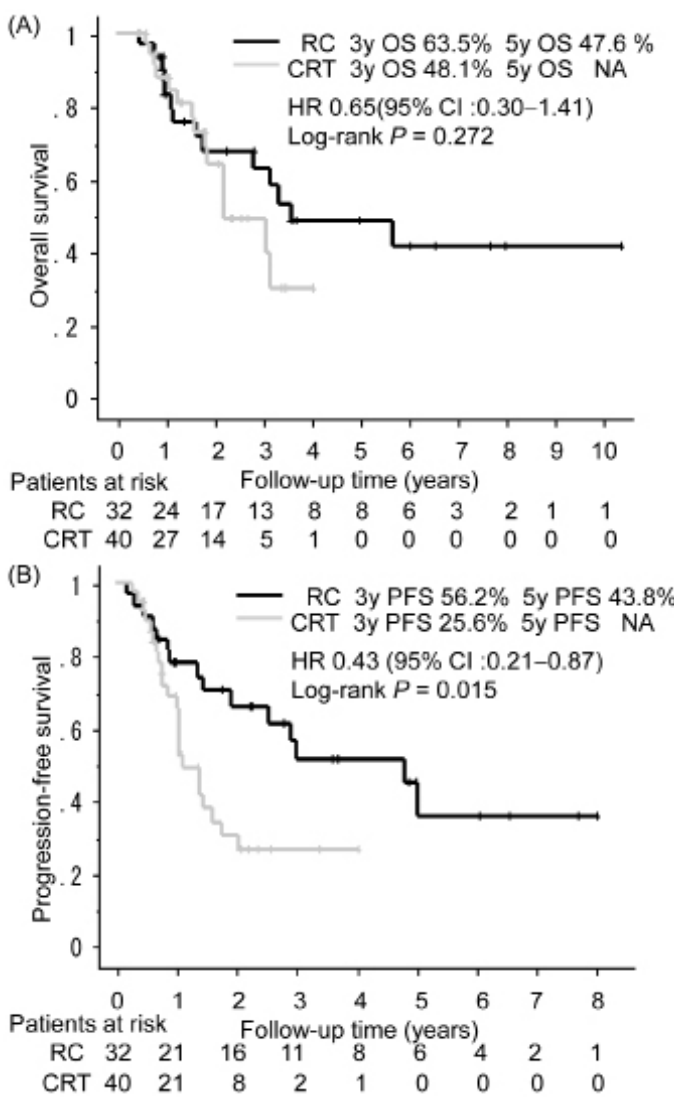

Figure 1. Kaplan-Meier Plots for (A) OS Rates and (B) PFS Rates. HR, Hazard Ratio; CI, Confidence Interval; NA, not Assessed

Table 1. Patient Characteristics $(n=72)$

\begin{tabular}{lccc}
\hline Characteristic & $\begin{array}{c}\text { RC group } \\
(\mathrm{n}=32)\end{array}$ & $\begin{array}{c}\text { CRT group } \\
(\mathrm{n}=40)\end{array}$ & $\begin{array}{c}\mathrm{P} \\
\text { value }\end{array}$ \\
\hline $\begin{array}{l}\text { Age, years } \\
\text { median (range) }\end{array}$ & $68.6(44-82)$ & $71.0(61-82)$ & 0.225 \\
$\begin{array}{l}\text { Gender } \\
\text { male/female }\end{array}$ & $21 / 11$ & $27 / 13$ & 0.892 \\
$\begin{array}{l}\text { ECOG PS } \\
\text { 0/1/2 }\end{array}$ & $5 / 20 / 7$ & $7 / 23 / 10$ & 0.932 \\
$\begin{array}{l}\text { Clinical / Pathological T stage } \\
\text { T3/T4 }\end{array}$ & $21 / 11$ & $22 / 18$ & 0.441 \\
$\begin{array}{l}\text { Lymph node metastasis } \\
\text { positive/negative }\end{array}$ & $11 / 21$ & $18 / 22$ & 0.441 \\
$\begin{array}{l}\text { Histopathology } \\
\text { UC/non-UC }\end{array}$ & $29 / 3$ & $39 / 1$ & 0.610 \\
$\begin{array}{l}\text { Follow-up, months } \\
\text { median (range) }\end{array}$ & $27.2(6.0-124.8)$ & $19.5(5.3-48.5)$ & 0.018 \\
\hline
\end{tabular}

RC, radical cystectomy; CRT, chemoradiotherapy; ECOG PS, Eastern Cooperative Oncology Group performance status; UC, urothelial carcinoma; significant at $\mathrm{p}<0.05$

Table 2. Multivariate Cox Proportional Hazards Analysis for Effect of Radical Cystectomy (RC)

\begin{tabular}{|c|c|c|c|c|c|c|c|c|}
\hline \multirow{3}{*}{ Factor } & \multicolumn{3}{|c|}{ Overall survival } & \multicolumn{5}{|c|}{ Progression-free survival } \\
\hline & Model $1 \dagger$ & & Model 2末 & & Model $1 \dagger$ & & Model 2‡ & \\
\hline & HR (95\% CI) & $\mathrm{P}$ value & $\mathrm{HR}(95 \% \mathrm{CI})$ & $P$ value & HR $(95 \%$ CI $)$ & $P$ value & $\mathrm{HR}(95 \% \mathrm{CI})$ & $P$ value \\
\hline Age & $0.97(0.92-1.02)$ & 0.178 & $\mathrm{n} / \mathrm{d}$ & $\mathrm{n} / \mathrm{d}$ & $0.96(0.92-1.01)$ & 0.093 & $\mathrm{n} / \mathrm{d}$ & $\mathrm{n} / \mathrm{d}$ \\
\hline Gender & $1.19(0.48-2.93)$ & 0.708 & $\mathrm{n} / \mathrm{d}$ & $\mathrm{n} / \mathrm{d}$ & $1.17(0.56-2.44)$ & 0.681 & $\mathrm{n} / \mathrm{d}$ & $\mathrm{n} / \mathrm{d}$ \\
\hline ECOG PS & $1.67(0.68-4.10)$ & 0.260 & $\mathrm{n} / \mathrm{d}$ & $\mathrm{n} / \mathrm{d}$ & $1.50(0.65-3.45)$ & 0.345 & $\mathrm{n} / \mathrm{d}$ & $\mathrm{n} / \mathrm{d}$ \\
\hline Tumor stage & $2.13(0.95-4.74)$ & 0.066 & $\mathrm{n} / \mathrm{d}$ & $\mathrm{n} / \mathrm{d}$ & $3.91(1.91-7.99)$ & 0.001 & $\mathrm{n} / \mathrm{d}$ & $\mathrm{n} / \mathrm{d}$ \\
\hline Lymph node metastasis & $1.33(0.58-3.01)$ & 0.500 & $\mathrm{n} / \mathrm{d}$ & $\mathrm{n} / \mathrm{d}$ & $2.56(1.23-5.29)$ & 0.012 & $\mathrm{n} / \mathrm{d}$ & $\mathrm{n} / \mathrm{d}$ \\
\hline Treatment & $1.63(0.72-3.69)$ & 0.244 & $1.55(0.69-3.49)$ & 0.291 & $2.81(1.32-5.99)$ & 0.008 & $2.16(1.07-4.40)$ & 0.033 \\
\hline Propensity score & $\mathrm{n} / \mathrm{d}$ & $\mathrm{n} / \mathrm{d}$ & $0.93(0.08-10.1)$ & 0.952 & $\mathrm{n} / \mathrm{d}$ & $\mathrm{n} / \mathrm{d}$ & $3.67(0.38-35.1)$ & 0.259 \\
\hline
\end{tabular}

$\dagger$ Multivariable model 1 is adjusted for the effect of RC by controlling for age, gender, ECOG PS, tumor stage, lymph node metastasis, and treatment; $\ddagger$ Multivariable model 2 is adjusted for the effect of RC by applying a propensity score, which is a conditional probability of receiving RC given other factors including age, gender, ECOG PS, tumor stage, lymph node metastasis and treatment; ECOG PS, Eastern Cooperative Oncology Group performance status; HR, hazard ratio; CI, confidence interval; $\mathrm{n} / \mathrm{d}$, not done 
Table 3. Comparison of Adverse Events in The Radical Cystectomy and Chemoradiotherapy Groups

\begin{tabular}{lrr}
\hline Adverse event & $\begin{array}{c}\text { All grades } \\
\mathrm{n}(\%)\end{array}$ & $\begin{array}{r}\geq \text { Grade 3 } \\
\mathrm{n}(\%)\end{array}$ \\
\hline Radical cystectomy (n=48) & & \\
Pyelonephritis & $12(25.5)$ & $5(10.4)$ \\
Wound dehiscence & $7(14.6)$ & $1(2.1)$ \\
Paralytic ileus & $3(6.3)$ & \\
Adhesive ileus & $3(6.3)$ & $3(6.3)$ \\
Lymph leakage & $1(2.1)$ & \\
Acute coronary syndrome & $1(2.1)$ & \\
Stroke & $1(2.1)$ & \\
Intraoperative gastrointestinal injury & $1(2.1)$ & \\
Ureteric anastomotic leakage & $1(2.1)$ & $1(2.1)$ \\
Ureteric anastomotic stenosis & $1(2.1)$ & $1(2.1)$ \\
Urostomy stenosis & $1(2.1)$ & \\
Pelvic infection & $1(2.1)$ & \\
Chemoradiotherapy (n=43) & & \\
Anemia & $40(90.3)$ & $4(9.3)$ \\
Neutropenia & $42(97.7)$ & $35(81.4)$ \\
Thrombocytopenia & $25(58.1)$ & $6(14.0)$ \\
Febrile neutropenia & $6(14.0)$ & $6(14.0)$ \\
Nausea/vomiting & $29(67.4)$ & $2(4.7)$ \\
Diarrhea & $14(32.6)$ & \\
Cystitis & $10(23.3)$ & \\
Frequency & $19(44.2)$ & \\
Hematuria & $10(23.3)$ & $5(11.6)$ \\
\hline & & \\
& &
\end{tabular}

\section{Discussion}

In this retrospective study of patients with locally advanced bladder cancer, RC was a significant predictor of PFS but of OS. The 3-year PFS rate was $56.2 \%$ in the $\mathrm{RC}$ group and $25.6 \%$ in the CRT group ( $\mathrm{p}=0.015)$, whereas the 3 -year OS rates were $63.5 \%$ and $48.1 \%$, respectively $(\mathrm{p}=0.272)$.

There were many more grade 3 or higher adverse events in the CRT group than in the RC group, the majority of the adverse events in the former group occurred during the first cycle of MVAC. However, GU and GI toxicities were almost the same in the two groups.

There have been no randomized trials comparing the effects of RC and CRT treatment among patients with MIBC. Stein et al. recently reported that among 1,054 patients treated with RC for MIBC, the PFS and OS rates were $68 \%$ and $66 \%$ at 5 years, and $60 \%$ and $43 \%$ at 10 years, respectively (Stein et al., 2001). These investigators also reported that the 5-year PFS and OS rates for non-organ-confined (extravesical) lymph-nodenegative bladder cancer (pT3b, pT4) were 58\% and 47\%, respectively. Hautmann et al reported OS rates of 57.9\% and $44.3 \%$ at 5 and 10 years, respectively, among 1100 patients with UC who underwent RC with pelvic lymph node dissection without neoadjuvant therapy (Hautmann et al., 2012). These investigators also reported 5- and 10year disease-specific survival rates of $65.8 \%$ and $59.7 \%$ for patients with pT3N0 tumors and $46.1 \%$ and $36.5 \%$ for patients with pT4N0 tumors, respectively. Madersbacher and Studer et al reported that the PFS and OS rates at 5 years were $62 \%$ and $59 \%$, respectively, and $50 \%$ and $37 \%$ at 10 years; the 5 -year PFS rate was $52 \%$ for patients with pT3 tumors, and 36\% with for patients with pT4 tumors
(Madersbacher et al., 2003). In patients with MIBC treated with RC in Japan (Nishiyama et al., 2004), the 5-year OS rates for patients with stage pT2 or higher tumors were $46.3 \%$ in UC patients and $42.1 \%$ in non-UC patients, respectively. In our study, the 5-year OS rate for patients with locally advanced bladder cancer (T3-4a, N0 or N+, M0) who did not receive neoadjuvant chemotherapy was $47.6 \%$. Although our results are consistent with the results of the above-mentioned Japanese study, extravesical cancer is more life-threatening in Japan than in Europe or North America.

Shipley et al. reported the use of cisplatin combined with EBRT for the treatment of MIBC (clinical stages T2 to T4) in 1987 (Shipley et al., 1987). Since then the various CRT protocols for MIBC have been improved. Generally, the complete response rate is $59 \%$ to $72 \%$, and the OS rate is similar to that of RC for patients with MIBC. A multimodality bladder-sparing approach in a large series of patients has been reported by the University of Erlangen (Rodel et al., 2002), Massachusetts General Hospital (Efstathiou et al., 2012; Kachnic et al., 1997), the Radiation Therapy Oncology Group (Shipley et al., 1998; Kaufman et al., 2009) and the Bladder Cancer 2001 (BC2001) trial in the United Kingdom (James et al., 2012). The first three groups of patients received combined cisplatin-based chemotherapy and the Bladder Cancer 2001 chemotherapy regimen consisted of fluorouracil and mitomycin $\mathrm{C}$. The 5-year OS rates range from $48 \%$ to $52 \%$, but in patients with locally advanced cancer (T3 to T4), the 5-year OS was only 17\%-41\% (Rodel et al., 2002; Efstathiou et al., 2012; Shipley et al., 1998; James et al., 2012). In our study showed that the 3 -year OS rate for MVAC with EBRT was $48.1 \%$. Although the follow-up period in our study was shorter than that of these previous studies, CRT performed in patients with locally advanced bladder cancer revealed almost the same treatment effect.

Gemcitabine is a new drug used for many kinds of malignancies, and the combination of gemcitabine and cisplatin is a standard systemic chemotherapy for metastatic UC. Gemcitabine, a potent radiosensitizing agent, may be a candidate for a combined integrated approach to the treatment of MIBC. Concurrent weekly gemcitabine with EBRT has been tested in phase I studies (Borut et al., 2012; Oh et al., 2009; Caffo et al., 2003). Caffo et al. administered cisplatin at $100 \mathrm{mg} / \mathrm{m}^{2}$ on days 1 and 21 with weekly gemcitabine at $400 \mathrm{mg} / \mathrm{m}^{2}$ and concurrent 54 Gy EBRT in patients with bladder cancer staged pT2 or higher and without metastasis; the 5-year OS and PFS rates were $70.1 \%$ and $78.9 \%$, respectively (Caffo et al., 2003). In phase I studies, other investigators tested concurrent low-dose gemcitabine $\left(27 \mathrm{mg} / \mathrm{m}^{2}\right.$ twice weekly or $75 \mathrm{mg} / \mathrm{m}^{2}$ per week) with $60 \mathrm{~Gy} / 6$ weeks EBRT in patients with locally advanced bladder cancer without metastasis (Borut et al., 2012; Oh et al., 2009) and reported 5-year OS and PFS rates of $76 \%$ and $82 \%$, respectively. Borut et al. reported that the rates of grade 3 myelosuppression, GU toxicity and GI toxicity were $5 \%, 10 \%$ and $5 \%$, respectively (Borut and Lijana, 2012). Whereas these results showed relatively high performance in terms of treatment effect compared to cisplatin-based CRT, new chemotherapeutic agents would be contributed 
to choosing the treatment modalities of CRT in patients with locally advanced bladder cancer.

Although the survival rates of MIBC patients treated with CRT in previous trials are comparable to the rates for the RC series, this therapeutic option has not yet been established as the standard of care for MIBC. One reason is the lack of a randomized trial of this option. The Selective Bladder Preservation against Radical Excision (SPARE) trial randomized MIBC patients to confirm the effects of treatment with either RC or CRT was launched in the United Kingdom in 2007. However, the trial was prematurely closed because patient recruitment was hampered by the fact that the trial compared disparate treatment strategies (Paramasivan et al., 2011; Huddart et al., 2010). In contrast, in our study, there were no differences in patient background between the two groups, and $\mathrm{RC}$ resulted in significantly prolonged PFS compared to CRT, but the OS rates were similar in the two groups.

Previously reported CRT protocols vary enough that simple comparisons of adverse events are difficult. In our CRT group, grade 2 or higher myelosuppression was frequently observed, but GU and GI toxicities were similar in the two groups. In the Bladder Cancer 2001 trial, grade 3 or 4 adverse events occurred in $36 \%$ of patients in the CRT group; specifically, the rates of grade 2 or higher GU and GI toxicities in the CRT group were $21.3 \%$ and $9.6 \%$, respectively (James et al., 2012). In trials of cisplatin-based CRT, grade 3 or 4 leucopenia occurred in $16 \%$ to $32 \%$ of the patients (Rodel et al., 2002; Shipley et al., 1998). In a study of combined-modality treatment with selective organ preservation, Rodel et al., three (2\%) patients underwent cystectomy because of a shrinking bladder, six $(1.5 \%)$ patients required surgical intervention due to bowel obstruction and five $(3 \%)$ patients experienced reduced bladder capacity $(<2$-h micturition interval) (Rodel et al., 2002). In a study of 1013 ileal neobladder patients, Hautmann et al. reported various complications within the first 90 days of RC (Hautmann et al., 2010). Infectious complications were most common (24\%) followed by GU (17\%), GI (15\%) and wound-related complications (9\%); $22 \%$ of the patients had grade 3 to 5 complications, and the 90-day mortality rate was $2.3 \%$. In a study of complications after RC, Novara et al. reported that $13 \%$ of patients experienced grade 3 to 5 complications, and the 3-month mortality rate was 3\% (Novara et al., 2009). In our study, severe adverse events were rare in both groups, suggesting that both treatment options are acceptable for locally advanced bladder cancer, at least with regard to adverse events.

The potential limitations of our study need to be considered. First, our results were evaluated retrospectively, and the subjects were not randomized. Therefore, direct comparison between RC and CRT was difficult because of the potential for selection bias in retrospective studies and because of confounding resulting from discordance between clinical and pathological staging. Clinical staging errors in patients with invasive bladder cancer have been reported to occur in $30 \%-50 \%$ of patients (Stein et al., 2001; Teramukai et al., 2006). However, we observed no differences between the patient populations in the two groups. Second, our sample size was small, and the follow- up period was relatively short. The short follow-up period may have resulted in failure to evaluate late complications in the CRT group. Finally, we used the MVAC regimen, rather than gemcitabine and cisplatin, for concurrent CRT in this study. In our institution, the MVAC regimen has been the first-line treatment for advanced or metastatic $\mathrm{UC}$, and a gemcitabine and paclitaxel regimen is used as a second-line treatment, particularly for MVAC refractory patients previously treated effectively with MVAC (Ikeda et al., 2011).

\section{References}

Baykara M, Buyukberber S, Ozturk B, et al (2013). Efficacy and safety of concomitant chemoradiotherapy with cisplatin and docetaxel in patients with locally advanced squamous cell head and neck cancers. Asian Pac J Cancer Prev, 14, 2557-61.

Borut K, Lijana ZK (2012). Phase I study of radiochemotherapy with gemcitabine in invasive bladder cancer. Radiother Oncol, 102, 412-5.

Caffo O, Fellin G, Graffer U, et al (2003). Phase I study of gemcitabine and radiotherapy plus cisplatin after transurethral resection as conservative treatment for infiltrating bladder cancer. Int J Radiat Oncol Biol Phys, 57, 1310-6.

Cepeda MS, Boston R, Farrar JT, Strom BL (2003). Comparison of logistic regression versus propensity score when the number of events is low and there are multiple confounders. Am J Epidemiol, 158, 280-7.

Coppin CM, Gospodarowicz MK, James K, et al (1996). Improved local control of invasive bladder cancer by concurrent cisplatin and preoperative or definitive radiation. The National Cancer Institute of Canada Clinical Trials Group. J Clin Oncol, 14, 2901-7.

Efstathiou JA, Spiegel DY, Shipley WU, et al (2012). Long-term outcomes of selective bladder preservation by combinedmodality therapy for invasive bladder cancer: the MGH experience. Eur Urol, 61, 705-11.

Grossman HB, Natale RB, Tangen CM, et al (2003). Neoadjuvant chemotherapy plus cystectomy compared with cystectomy alone for locally advanced bladder cancer. $N$ Engl J Med, 349, 859-66.

Hashemi FA, Akbari EH, Kalaghchi B, Esmati E (2013). Concurrent chemoradiation with weekly gemcitabine and cisplatin for locally advanced cervical cancer. Asian Pac J Cancer Prev, 14, 5385-9.

Hautmann RE, Gschwend JE, de Petriconi RC, Kron M, Volkmer BG (2006). Cystectomy for transitional cell carcinoma of the bladder: results of a surgery only series in the neobladder era. J Urol, 176, 486-92.

Hautmann RE, de Petriconi RC, Volkmer BG (2010). Lessons learned from 1,000 neobladders: the 90-day complication rate. J Urol, 184, 990-4.

Hautmann RE, de Petriconi RC, Pfeiffer C, Volkmer BG (2012). Radical cystectomy for urothelial carcinoma of the bladder without neoadjuvant or adjuvant therapy: long-term results in 1100 patients. Eur Urol, 61, 1039-47.

Huddart RA, Hall E, Lewis R, Birtle A (2010). Life and death of spare (selective bladder preservation against radical excision): reflections on why the spare trial closed. $B J U$ Int, 106, 753-5.

Ikeda M, Matsumoto K, Niibe Y, et al (2011). The radiotherapy with methotrexate, vinblastine, doxorubicin, and cisplatin treatment is an effective therapeutic option in patients with advanced or metastatic bladder cancer. J Radiat Res, 52, 
674-9.

Ikeda M, Matsumoto K, Tabata K, et al (2011). Combination of gemcitabine and paclitaxel is a favorable option for patients with advanced or metastatic urothelial carcinoma previously treated with cisplatin-based chemotherapy. Jpn J Clin Oncol, 41, 1214-20.

Jemal A, Bray F, Center MM, et al (2011). Global cancer statistics. CA Cancer J Clin, 61, 69-90.

James ND, Hussain SA, Hall E, et al (2012). Radiotherapy with or without chemotherapy in muscle-invasive bladder cancer. N Engl J Med, 366, 1477-88.

Kachnic LA, Kaufman DS, Heney NM, et al (1997). Bladder preservation by combined modality therapy for invasive bladder cancer. J Clin Oncol, 15, 1022-9.

Kaufman DS, Winter KA, Shipley WU, et al (2009). Phase I-II RTOG study (99-06) of patients with muscle-invasive bladder cancer undergoing transurethral surgery, paclitaxel, cisplatin, and twice-daily radiotherapy followed by selective bladder preservation or radical cystectomy and adjuvant chemotherapy. Urology, 73, 833-7.

Kilic D, Yalman D, Aksu G, et al (2012). Impact of adjuvant chemoradiotherapy for rectal cancer on the long-term quality of life and late side effects: a multicentric clinical evaluation by the Turkish Oncology Group. Asian Pac J Cancer Prev, 13, 5741-6.

Liang ZG,Zhu XD, Tan AH, et al (2013). Induction chemotherapy followed by concurrent chemoradiotherapy versus concurrent chemoradiotherapy with or without adjuvant chemotherapy for locoregionally advanced nasopharyngeal carcinoma: meta-analysis of 1,096 patients from 11 randomized controlled trials. Asian Pac J Cancer Prev, 14, 515-21.

Madersbacher S, Hochreiter W, Burkhard F, et al (2003). Radical cystectomy for bladder cancer today - a homogeneous series without neoadjuvant therapy. J Clin Oncol, 21, 690-6.

Nishiyama H, Habuchi T, Watanabe J, et al (2004). Clinical outcome of a large-scale multi-institutional retrospective study for locally advanced bladder cancer: a survey including 1131 patients treated during 1990-2000 in Japan. Eur Urol, 45, 176-81.

Novara G, De Marco V, Aragona M, et al (2009). Complications and mortality after radical cystectomy for bladder transitional cell cancer. $J$ Urol, 182, 914-21.

Oh KS, Soto DE, Smith DC, et al (2009). Combined-modality therapy with gemcitabine and radiation therapy as a bladder preservation strategy: long-term results of a phase I trial. Int J Radiat Oncol Biol Phys, 74, 511-7.

Paramasivan S, Huddart R, Hall E, et al (2011). Key issues in recruitment to randomised controlled trials with very different interventions: a qualitative investigation of recruitment to the SPARE trial (CRUK/07/011). Trials, 12,78 .

Rodel C, Grabenbauer GG, Kuhn R, et al (2002). Combinedmodality treatment and selective organ preservation in invasive bladder cancer: long-term results. J Clin Oncol, 20, 3061-71.

Shipley WU, Prout GR, Jr Einstein AB, et al (1987). Treatment of invasive bladder cancer by cisplatin and radiation in patients unsuited for surgery. JAMA, 258, 931-5.

Shipley WU, Winter KA, Kaufman DS, et al (1998). Phase III trial of neoadjuvant chemotherapy in patients with invasive bladder cancer treated with selective bladder preservation by combined radiation therapy and chemotherapy: initial results of Radiation Therapy Oncology Group 89-03. J Clin Oncol, 16, 3576-83.

Stein JP, Lieskovsky G, Cote R, et al (2001). Radical cystectomy in the treatment of invasive bladder cancer: long-term results in 1,054 patients. J Clin Oncol, 19, 666-75.
Sternberg CN, Yagoda A, Scher HI, et al (1985). Preliminary results of M-VAC (methotrexate, vinblastine, doxorubicin and cisplatin) for transitional cell carcinoma of the urothelium. J Urol, 133, 403-7.

Teramukai S, Nishiyama H, Matsui Y, Ogawa O, Fukushima $M$ (2006). Evaluation for surrogacy of end points by using data from observational studies: tumor downstaging for evaluating neoadjuvant chemotherapy in invasive bladder cancer. Clin Cancer Res. 12, 139-43.

von der Maase H, Hansen SW, Roberts JT, et al (2000). Gemcitabine and cisplatin versus methotrexate, vinblastine, doxorubicin, and cisplatin in advanced or metastatic bladder cancer: results of a large, randomized, multinational, multicenter, phase III study. J Clin Oncol, 18, 3068-77.

Yu HS, Wang X, Song AQ, et al (2012). Concurrent chemoradiotherapy versus radiotherapy alone for locoregionally advanced nasopharyngeal carcinoma. Asian Pac J Cancer Prev. 13, 3961-5. 